\title{
Simulating Site-Specific Effects of a Changing Climate on Jack Pine Productivity Using a Modified Variant of the CROPLANNER Model
}

\author{
Peter F. Newton \\ Canadian Wood Fibre Centre, Canadian Forest Service, Natural Resources Canada, Ontario, Canada \\ Email: peter.newton@nrcan.gc.ca
}

Received September 17 ${ }^{\text {th }}, 2011$; revised October $29^{\text {th }}, 2011$; accepted November $8^{\text {th }}, 2011$

\begin{abstract}
This study evaluated the site-specific effects of projected future climate conditions on the productivity of jack pine (Pinus banksiana Lamb.) plantations over the next 50 years (2011-2061). Climatic parameters as predicted by the Canadian Global Climate Model in association with a regional spatial climatic model, under 3 emissions scenarios (no change (NC), B1 and A2), were used as input values to a biophysicalbased site-specific height-age model that was integrated into the CROPLANNER model and associated algorithm. Plantations managed under a basic silvicultural intensity on two site qualities at each of two geographically separated sites (northeastern and northwestern Ontario, Canada) were assessed. The results indicated that the stands situated on low-to-medium quality sites at both locations were largely unaffected by the predicted increase in temperature and precipitation rates. Conversely, however, stands situated on good-to-excellent quality sites grown under the B1 and A2 scenarios experienced consequential declines in stand development rates resulting in decreases in rotational mean sizes, biomass yields, recoverable end-product volumes, and economic worth. In addition to providing a plausible range of site-specific climate change outcomes on jack pine productivity within the central portion of the species range, these results suggest that future predictions that do not account for potential climate changes effects may overestimate merchantable productivity on the higher site qualities by approximately $15 \%$. As demonstrated, incorporating biophysical-based site index functions within existing forest productivity models may represent a feasible approach when accounting for climate change effects on yield outcomes of boreal species.
\end{abstract}

Keywords: B1 and A2 Emission Scenarios; Low-to-Medium and Good-to-Excellent Site Qualities; Basic Silvicultural Intensity Regimes

\section{Introduction}

Jack pine (Pinus banksiana Lamb.) occupies a wide range of sites throughout the northern temperate forest region of North America (Rudolph \& Yeatman, 1982). Ecologically, jack pine exhibits an ability to: 1) tolerate environment constraints (e.g., moisture and nutrient limitations); 2) temporarily exploit environments following severe disturbance (e.g., rapidly colonization of recently-disturbed wildfire-burned sites due to its early maturity (cone-bearing at 3 - 5 years of age), cone serotiny and associated seed-dispersal habit (aerial dispersal of abundant viable seeds following heat-induced opening of serotinous cones), and favorable germination and seedling establishment conditions following wildfire (e.g., exposed mineral soil and temporary lack of competing species)); and 3) intensely compete for environmental resources and/or physical space, as evident by its asymmetric size-distributions and rapid rates of self-thinning as observed within monospecific density-stressed populations. Jack pine is one of the most economically important and intensely managed species within the Canadian Boreal Forest Region (Rowe, 1972). Specifically, in Canada's largest province, Ontario, jack pine represents $33 \%$ of the annual softwood volume harvested (OMNR, 2008). The principal products derived from this harvest are northern bleached softwood kraft pulp used to produce paperboard, tissue products and newsprint, dimensional lumber (studs, structural joists and planks), and composite wood products that are used in wood-frame residential house construction (Zhang \& Koubaa, 2008).

The effects of a changing climate on boreal ecosystems have been estimated using a variety of different modeling approaches (e.g., Myneni et al. (1997), Shaw et al. (2006), Girardin et al. (2008), Kurz et al. (2009), Ise and Moorcroft (2010)). Although contradictory tendencies have been reported, results from these analyses have been useful in delineating a range of plausible outcomes. At the regional level, recognition that the jack pine resource will be impacted by rapid changes in climate is well appreciated throughout the forest management and scientific communities (e.g., Parker et al., 2000; Colombo et al., 2007). Although climate change projections vary by the type of model used, emissions scenario considered, and locality assessed, the consensus is that the climate within northeastern and northwestern Ontario will undergo a consequential change over the next century (Colombo et al., 2007). Given that jack pine exhibits a large degree of phenotypic plasticity and has the ability to tolerate a wide range of climatic and site conditions, it is important to estimate how this valuable resource will fair under a changed climate. Consequently, the objectives of this study were to simulate climate change effects on the productivity of jack pine plantations across a range of site qualities and locations. The approach employed the CROPLANNER decision-support model (Newton, 2009) which was modified through the incorporation of a bio- 
physical-based site-specific height-age function. This enabled an empirical assessment of the effects of localized changes in climate on jack pine productivity.

Briefly, CROPLANNER is an algorithmic analogue of the structural stand density management model (SSDMM) which was developed by expanding the stand density management diagram modelling framework through the incorporation of distribution recovery modules for diameter, height, biomass, carbon, end-products, value and wood quality attributes (Newton, 2009). Structurally, the model consists of a number of functional and empirical quantitative relationships, which collectively represent the cumulative effect of various underlying competition processes on tree and stand yield parameters. The temporal dependency of these processes is governed by the intensity of competition and site quality as expressed by relative density index and site index, respectively. As with most tree and stand simulators, the site-specific mean dominant height-age function largely governs the rate of stand development. Consequently, accounting for the cumulative effect of climate change through the use of a biophysical-based site-specific height-age function was considered a prudent approach and is in accord with similar approaches used with other tree and stand simulators (e.g., O'Neill \& Nigh, 2011).

CROPLANNER, for a given density management regime, site quality, rotation length, cost profile and set of merchantability specifications, enables the estimation of overall productivity (e.g., mean annual volume, biomass and carbon increments), volumetric yields (e.g., total and merchantable volumes per unit area), log-product distributions (e.g., number of pulp and saw logs), biomass production and carbon sequestration outcomes (e.g., oven-dried masses of above-ground components and associated carbon equivalents), recoverable end-products and associated monetary values (e.g., volume and economic value of recovered chip and dimensional lumber products) by sawmill-type (stud and randomized length processing protocols), economic efficiency (e.g., land expectation value), duration of optimal site occupancy, structural stability, and fibre attributes (e.g., wood density and branch diameter). For a complete analytical description of the approach used in the development and calibration of the modular-based SSDMM, refer to Newton (2009).

The scope of this analysis includes the consideration of the effects of B1 and A2 emission scenarios (Nakicenovic et al., 2000) relative to the null case of no climate change (NC). These scenarios cover the plausible range normally considered by policy makers and forest planners within the Province of Ontario (Colombo et al., 2007). Specifically, the NC scenario was based on climatic norms observed during the 1970-2000 period. As defined by Nakicenovic et al. (2000), the B1 emission scenario arises from a convergent world that is characterized by global-based collaborative approaches to solving economic, social, and environmental problems (i.e., best-case scenario). The transition from conventional oil and gas resources to alternative energy systems is expected to be uneventful. Globally, the population is expected to increase to 8.7 billion by 2050 but then decline to 7.0 billion by 2100 . The global gross domestic product (GDP) is expected to increase to 136 billion (1990 US\$/yr) by 2050 and then to 328 billion (1990 US\$/yr) by 2100 . Proactive nation-based environmental measures and associated policies are expected to result in relatively low anthropogenic GHGs and aerosols emissions, reaching a maximum concentration of approximately 600 ppm by 2100 (IPPC, 2007). Relative to the 1980-2000 period, the mean global temperature is ex- pected to increase $2.4^{\circ} \mathrm{C}$ by 2100 (IPPC, 2007).

Conversely, the A2 emission scenario is based on a hetero- geneous and regionally differentiated world in terms of econo- mic development, productivity, social structures and income equa- lity, where the self-reliance and preservation of local identities is paramount (i.e., worst-case scenario; Nakicenovic et al., 2000). Although attempts to control local environmental degradation will occur, international co-operation in mitigating the effects of climate change will be limited. The global population is expected to increase to 11.3 billion by 2050 and then to 15.1 billion by 2100 . Global GDP is projected to increase to 82 billion (1990 US\$/yr) by 2050 and then to 243 billion (1990 US\$/yr) by 2100 . This increase in population growth combined with concurrent changes in land use, and consumption of natural resources including the rapid depletion of fossil fuels, will substantially increase GHGs and aerosols emissions, reaching a maximum concentration of approximately 1250 ppm by 2100 (IPPC, 2007). Resultantly, the mean global temperature is expected to increase by approximately $3.4^{\circ} \mathrm{C}$ by 2010 , relative to the $1980-2000$ period (IPPC, 2007).

\section{Material and Methods}

Firstly, the CROPLANNER decision-support model (Newton, 2009) was modified through the inclusion of a biophysicalbased height-age model developed for jack pine plantations in Ontario (Sharma et al., 2012). Specifically, the original site-based height-age function developed by Carmean et al. (2001; Equation (23) in Newton, 2009) was replaced by Equation (1):

$$
H=1.3+\hat{\beta}_{0} /\left(1-\left(1-\left(\hat{\beta}_{0} / S_{I}\right)\right)\left(25 /\left(A_{b h}-0.5\right)\right)^{\hat{\beta}_{1}}\right)
$$

where

$$
\begin{aligned}
& \hat{\beta}_{0}=196.47+0.3209 P_{w}-0.3762 P_{d}-14.2779 T_{g}+0.0014 P_{a} T_{a}, \\
& \hat{\beta}_{1}=-1.3757-0.0058 P_{w}+0.0114 P_{d}+0.2153 T_{g}
\end{aligned}
$$

$H(\mathrm{~m})$ is mean dominant height, $S_{I}$ is site index (mean dominant height at a breast-height age of $25 \mathrm{yr}$ ), $A_{b h}$ is mean breast height age $(\mathrm{yr}), P_{w}$ and $P_{d}$ are total precipitation $(\mathrm{mm})$ during the wettest and driest period, respectively, $T_{g}$ is the mean temperature $\left({ }^{\circ} \mathrm{C}\right)$ during the growing season, $P_{a}$ is the total precipitation $(\mathrm{mm})$ during the entire year, and $T_{a}$ is the mean annual temperature $\left({ }^{\circ} \mathrm{C}\right)$ during the entire year.

Secondly, climatic parameters $\left(P_{w}, P_{d}, T_{q}, P_{a}\right.$ and $\left.T_{a}\right)$ were predicted by the Canadian Global Climate Model (V. 3.1; Environment Canada, 2011) in association with a regional spatial climatic model (McKenney et al., 2007). Two emissions scenarios (B1 and A2; Nakicenovic et al., 2000) for the 2011-2040 and 2041-2070 periods at two geographic locations (Kirkland Lake, ON (Forest Section B7 Missinaibi-Cabonga (Rowe, 1972)) and Thunder Bay, ON (B.9 Superior (Rowe, 1972))) were specified. For the no change scenario (NC), corresponding climatic variables for the 1970-2000 period were obtained from the regional spatial climatic model (McKenney et al., 2007) for the same locations. The 2011-2040 and 2041-2070 values were combined and means obtained for the entire 2011-2070 period in order to avoid abrupt changes at the 2040-2041 transition point. Table 1 lists the derived parameters for each location by scenario and time period.

Thirdly, the simulations consisted of inputting the resultant climatic values into the modified CROPLANNER model and implementing density management regimes consistent with a basic silvicultural intensity (Bell et al., 2008) on poor-to-medium 


\section{P. F. NEWTON}

Table 1.

Climatic input parameters for the modified CROPLANNER model: climatic variables corresponding to the NC, B1 and A2 emission scenarios by period and location.

\begin{tabular}{|c|c|c|c|c|c|c|c|c|c|c|c|c|c|c|}
\hline \multirow{4}{*}{$\begin{array}{l}\text { Input } \\
\text { Parameter } \\
\text { [Denotation] }\end{array}$} & \multicolumn{14}{|c|}{ Location (Forest Section) $^{\mathrm{a}}$} \\
\hline & \multicolumn{7}{|c|}{ Kirkland Lake, ON (B.7 Missinaibi-Cabonga) } & \multicolumn{7}{|c|}{ Thunder Bay, ON (B.9 Superior) } \\
\hline & $\mathrm{NC}$ & & B1 & & & $\mathrm{A} 2$ & & $\mathrm{NC}$ & & $\mathrm{B} 1$ & & & $\mathrm{~A} 2$ & \\
\hline & 1971 & 2011 & 2041 & 2011 & 2011 & 2041 & 2011 & 1971 & 2011 & 2041 & 2011 & 2011 & 2041 & 2011 \\
\hline & 2000 & 2040 & 2070 & 2070 & 2040 & 2070 & 2070 & 2000 & 2040 & 2070 & 2070 & 2040 & 2070 & 2070 \\
\hline $\begin{array}{l}\text { Total precipitation during wettest } \\
\text { period }(\mathrm{mm})\left[P_{\mathrm{w}}\right]\end{array}$ & 98 & 99 & 98 & 99 & 107 & 100 & 104 & 87 & 91 & 91 & 91 & 97 & 87 & 92 \\
\hline $\begin{array}{l}\text { Total precipitation during driest } \\
\text { period }(\mathrm{mm})\left[P_{d}\right]\end{array}$ & 47 & 49 & 59 & 54 & 61 & 59 & 60 & 28 & 32 & 38 & 35 & 38 & 36 & 37 \\
\hline $\begin{array}{l}\text { Mean temperature during growing } \\
\text { season }\left({ }^{\circ} \mathrm{C}\right)\left[T_{g}\right]\end{array}$ & 11.2 & 12.5 & 13.6 & 13.1 & 12.9 & 13.8 & 13.4 & 11.3 & 12.7 & 13.6 & 13.2 & 13.1 & 14.0 & 13.6 \\
\hline Annual precipitation $(\mathrm{mm})\left[P_{a}\right]$ & 865 & 885 & 951 & 918 & 951 & 964 & 958 & 720 & 765 & 816 & 791 & 811 & 811 & 811 \\
\hline Annual mean temperature $\left({ }^{\circ} \mathrm{C}\right)\left[T_{a}\right]$ & 1.54 & 2.71 & 4.01 & 3.36 & 3.33 & 4.85 & 4.09 & 2.74 & 3.98 & 5.25 & 4.62 & 4.50 & 6.14 & 5.32 \\
\hline
\end{tabular}

All forecasted values of the climatic parameters were derived from the Canadian Global Climate Model (Version 3.1; Environment Canada, 2011). Specific estimates for

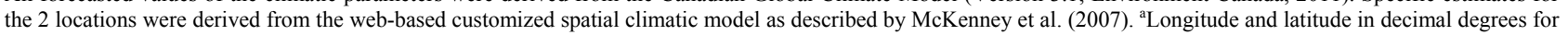
Kirkland Lake, ON and Thunder Bay, ON: -80.0333 and 48.1500, -89.2500 and 48.3833, respectively. Forest Section after Rowe (1972).

and good-to-excellent sites within each of the two locales. The underlying objective of the basic silvicultural intensity is to shorten the rotation length and increase product value. The actual crop plan consisted of site preparation and vegetation management treatments preceding the planting of 2000 seedlings per hectare and allowing 500 seedlings per hectare to establish naturally as ingress. Table 2 lists the input variables and model parameters that were used.

\section{Results}

During the 2011-2070 period, both precipitation and temperatures are predicted to increase at both the eastern and western Ontario locations with the greatest increases occurring under the A2 emission scenario (Table 1). Specifically, relative to the 1971-2000 period (Table 1): 1) annual precipitation is forecasted to increase $6.1 \%$ and $10.8 \%$ and mean annual temperatures by $+1.8^{\circ} \mathrm{C}$ and $+2.6^{\circ} \mathrm{C}$ under the $\mathrm{B} 1$ and $\mathrm{A} 2$ scenarios, respectively, at the northeastern locale; and 2) annual precipitation is forecasted to increase $9.9 \%$ and $12.6 \%$ and mean annual temperatures by $+1.9^{\circ} \mathrm{C}$ and $+2.6^{\circ} \mathrm{C}$ under the $\mathrm{B} 1$ and $\mathrm{A} 2$ scenarios, respectively, at the northwestern locale. Simulating the effect of these increases on jack pine plantations established at nominal initial spacing across a range of site qualities using the CROPLANNER model (Table 2), enabled a comparative analysis of $50 \mathrm{yr}$ rotational productivity outcomes for each emission scenario. Table 3(a) and Table 3(b) list the yield estimates for the northeastern and northwestern locales, respectively. Similarly, Tables 4(a) and (b) provide a set of relevant performance metrics for the northeastern and northwestern locales, respectively. Site-specific representations of the expected sizedensity trajectories within the traditional stand density management graphic for each scenario by site quality and geographic location are shown in Figures 1 and 2: Figures 1(a) and (b) illustrate the trajectories for the poor-to-medium and good-to-excellent site qualities at the north-eastern locale, respectively, whereas Figures 2(a) and (b) illustrate the corresponding trajectories at the northwestern locale, respectively.
Table 2.

Density management input parameters for the modified CROPLANNER model.

\begin{tabular}{|c|c|}
\hline Parameter (units) & Value \\
\hline Simulation Year & 2011 \\
\hline Site Index $\left(S_{I}\right)$ & $8 \& 12$ \\
\hline Rotation Age (yr) & 50 \\
\hline Initial Density (stems/ha) & 2500 \\
\hline Ingress Density (stems/ha) & 500 \\
\hline Pulp Log Length (m) & 2.59 \\
\hline Pulp Log Diameter $(\mathrm{cm})$ & 10 \\
\hline Saw Log Length (m) & 5.03 \\
\hline Saw Log Diameter $(\mathrm{cm})$ & 14 \\
\hline Merchantable Top Diameter (cm) & 4 \\
\hline Inflation Rate $(\%)$ & 2 \\
\hline Discount Rate (\%) & 4 \\
\hline $\begin{array}{l}\text { Operability Target for } S_{I}=8: \\
\text { Quadratic Mean Diameter }(\mathrm{cm})\end{array}$ & 14 \\
\hline $\begin{array}{l}\text { Operability Target for } S_{I}=12 \text { : } \\
\text { Quadratic Mean Diameter }(\mathrm{cm})\end{array}$ & 18 \\
\hline Site Preparation (CAN\$/ha) & 300 \\
\hline Planting (CAN\$/seedling) & 0.6 \\
\hline Operational Adjustment Factor (\%) & 1 \\
\hline Product Degrade Factor (\%) & 15 \\
\hline $\begin{array}{l}\text { Harvesting }+ \text { Stumpage }+ \text { Renewal }+ \\
\text { Transportation }+ \text { Manufacturing Costs }\left(\mathrm{CAN} \$ / \mathrm{m}^{3}\right)\end{array}$ & 75 \\
\hline
\end{tabular}




\section{P. F. NEWTON}

\section{Lower Site Qualities}

The stands established on the poor-to-medium site qualities differed only slight in terms of their dynamics over the $50 \mathrm{yr}$ rotations (Figures 1(a) and 2(a)). However, for the northeastern stands, these slight differences in the size-density trajectories translated into greater rotational mean sizes (quadratic mean diameter and mean volume) and per unit area yields (total and merchantable volumes, and component-specific biomass and carbon yields), and end-product volumes (volume of recoverable chip and lumber volumes), for stands grown under the NC and B1 scenarios, relative to the stand grown under the A2 scenario (Table 3(a)). Conversely, however, the trajectories for the northwestern stands grown under the B1 and A2 scenarios actually attained a slightly greater size-density condition at rotation than that obtained within the stand grown under the NC scenario. These differences translated into greater rotational mean sizes and per unit area yields, and preferred end-product volumes, relative to the NC scenario (Table 3(b)). In terms of the performance indices, merchantable volume, biomass productivity and carbon yields, and the proportion of preferred end-products (number of sawlogs and volume of recoverable dimension lumber), were slightly greater for northeastern stands growing under the $\mathrm{NC}$ and $\mathrm{B} 1$ scenarios relative to the stand grown under the A2 scenario (Table 4(a)). Apart from a decline in economic worth under the A2 scenario, differences in the duration of optimal site occupancy, stand structure, wood quality metrics, and time to operability, were largely inconsequential (Table 4(a)). The corresponding values for the northwestern stands established on a poor-to-medium site quality, indicated that productivity and the percentage of preferred end-products increased slightly under the B1 and A2 scenarios, resulting in a consequential increase in economic worth relative to the NC scenario (Table 4(b)). Similar to the northeastern stands, differences in the duration of optimal site occupancy, stand structure, wood quality metrics, and time to operability, were minimal. Although, the stand grown under the NC scenario exhibited a slight increase in its rate of development as evident by a $3 \mathrm{yr}$ differential (reduction) in the time to operability status (Table 4(b)).

Table 3.

(a) Rotational yield estimates for jack pine plantations managed under a basic silvicultural intensity in northeastern Ontario by emission scenario and site quality. (b) Rotational yield estimates for jack pine plantations managed under a basic silvicultural intensity in northwestern Ontario by emission scenario and site quality.

(a)

\begin{tabular}{|c|c|c|c|c|c|c|}
\hline \multirow{4}{*}{$\begin{array}{l}\text { Attribute }^{\mathrm{a}} \\
\text { (Unit) } \\
\end{array}$} & \multicolumn{6}{|c|}{ Site quality $^{\mathrm{b}}$} \\
\hline & \multicolumn{3}{|c|}{ Poor-to-medium } & \multicolumn{3}{|c|}{ Good-to-excellent } \\
\hline & \multicolumn{3}{|c|}{ Emission scenario $^{c}$} & \multicolumn{3}{|c|}{ Emission scenario $^{\mathrm{c}}$} \\
\hline & $\mathrm{NC}$ & $\begin{array}{c}\text { B1 vs NC } \\
(\% \Delta)\end{array}$ & $\begin{array}{c}\mathrm{A} 2 \text { vs NC } \\
(\% \Delta)\end{array}$ & $\mathrm{NC}$ & $\begin{array}{c}\text { B1 vs NC } \\
(\% \Delta)\end{array}$ & $\begin{array}{c}\mathrm{A} 2 \text { vs NC } \\
(\% \Delta)\end{array}$ \\
\hline Mean dominant height $(\mathrm{m})$ & 16.2 & 0.0 & -2.5 & 21.7 & -7.8 & -13.4 \\
\hline Quadratic mean diameter $(\mathrm{cm})$ & 15.9 & 0.0 & -4.4 & 23.1 & -10.4 & -16.9 \\
\hline Total basal area per stand $\left(\mathrm{m}^{2} / \mathrm{ha}\right)$ & 29 & 0.0 & -6.9 & 35 & -5.7 & -11.4 \\
\hline Mean volume per tree $\left(\mathrm{dm}^{3}\right)$ & 140 & 0.0 & -11.4 & 393 & -26.0 & -39.7 \\
\hline Total volume per stand $\left(\mathrm{m}^{3} / \mathrm{ha}\right)$ & 202 & 0.5 & -7.9 & 326 & -13.2 & -22.1 \\
\hline Total merchantable volume per stand $\left(\mathrm{m}^{3} / \mathrm{ha}\right)$ & 190 & 0.5 & -8.9 & 313 & -13.4 & -22.7 \\
\hline Total density per stand (stems/ha) & 1445 & 0.8 & 3.8 & 829 & 17.2 & 29.3 \\
\hline Relative stand density $(\% / 100)$ & 0.64 & 1.6 & -6.3 & 0.86 & -8.1 & -15.1 \\
\hline Total number of pulp logs per stand (logs/ha) & 2313 & 0.7 & -6.4 & 1550 & 35.7 & 47.5 \\
\hline Total number of saw logs per stand (logs/ha) & 172 & 0.6 & -31.4 & 1323 & -32.9 & -53.0 \\
\hline Total residual tip volume per stand $\left(\mathrm{m}^{3} / \mathrm{ha}\right)$ & 49.9 & 0.8 & -6.8 & 21.3 & 24.9 & 57.3 \\
\hline Total bark oven-dried biomass per stand (t/ha) & 10.9 & 0.9 & -5.5 & 12.1 & 0.0 & -1.7 \\
\hline Total stem oven-dried biomass per stand (t/ha) & 119.8 & 0.8 & -8.7 & 180.3 & -12.4 & -19.9 \\
\hline Total branch oven-dried biomass per stand ( $\mathrm{t} / \mathrm{ha}$ ) & 10.2 & 0.0 & -8.8 & 18.3 & -15.3 & -24.0 \\
\hline Total foliage oven-dried biomass per stand (t/ha) & 6.1 & 0.0 & -8.2 & 9.4 & -11.7 & -18.1 \\
\hline Total above-ground oven-dried biomass per stand ( $\mathrm{t} / \mathrm{ha})$ & 147.0 & 0.6 & -8.4 & 220.0 & -11.8 & -19.1 \\
\hline Total bark carbon per stand (t/ha) & 5.5 & 0.0 & -7.3 & 6.0 & 1.7 & 0.0 \\
\hline Total stem carbon per stand (t/ha) & 59.9 & 0.7 & -8.7 & 90.2 & -12.4 & -20.0 \\
\hline Total branch carbon per stand $(\mathrm{t} / \mathrm{ha})$ & 5.1 & 0.0 & -9.8 & 9.1 & -14.3 & -24.2 \\
\hline Total foliage carbon per stand (t/ha) & 3.0 & 0.0 & -6.7 & 4.7 & -10.6 & -19.1 \\
\hline Total above-ground carbon per stand (t/ha) & 73.5 & 0.5 & -8.4 & 110.0 & -11.8 & -19.2 \\
\hline Total chip volume per stand—stud mill $\left(\mathrm{m}^{3} / \mathrm{ha}\right)$ & 74.2 & 0.8 & -8.6 & 106.8 & -11.7 & -19.0 \\
\hline Total lumber volume per stand-stud mill $\left(\mathrm{m}^{3} / \mathrm{ha}\right)$ & 83.9 & 0.8 & -12.9 & 189.8 & -23.4 & -35.8 \\
\hline Total chip volume per stand-randomized length $\mathrm{mill}\left(\mathrm{m}^{3} / \mathrm{ha}\right)$ & 46.1 & 0.9 & -8.9 & 69.5 & -12.8 & -20.6 \\
\hline Total lumber volume per stand—randomized length $\mathrm{mill}\left(\mathrm{m}^{3} / \mathrm{ha}\right)$ & 112.6 & 0.8 & -11.5 & 227.2 & -21.1 & -32.4 \\
\hline
\end{tabular}

${ }^{a}$ Predicted values; ${ }^{\mathrm{b}}$ Poor-to-medium and good-to-excellent site qualities correspond to site indices of 8 and $12 \mathrm{~m}$ at a breast-height age of 25 yrs, respectively (Sharma et al., 2012); ${ }^{\mathrm{c}}$ As defined in the text. 


\section{P. F. NEWTON}

(b)

\begin{tabular}{|c|c|c|c|c|c|c|}
\hline \multirow{2}{*}{$\begin{array}{l}\text { Attribute }^{\mathrm{a}} \\
\text { (Unit) }\end{array}$} & \multicolumn{6}{|c|}{ Site quality ${ }^{\mathrm{b}}$} \\
\hline & \multicolumn{3}{|c|}{ Poor-to-medium } & \multicolumn{3}{|c|}{ Good-to-excellent } \\
\hline & \multicolumn{3}{|c|}{ Emission scenario $^{\mathrm{c}}$} & \multicolumn{3}{|c|}{ Emission scenario $^{c}$} \\
\hline & $\mathrm{NC}$ & $\begin{array}{c}\text { B1 vs NC } \\
(\% \Delta)\end{array}$ & $\begin{array}{c}\text { A2 vs NC } \\
(\% \Delta)\end{array}$ & $\mathrm{NC}$ & $\begin{array}{c}\text { B1 vs NC } \\
(\% \Delta)\end{array}$ & $\begin{array}{c}\text { A2 vs NC } \\
(\% \Delta)\end{array}$ \\
\hline Mean dominant height $(\mathrm{m})$ & 15.5 & 3.2 & 1.9 & 20.9 & -5.3 & -7.2 \\
\hline Quadratic mean diameter $(\mathrm{cm})$ & 15.0 & 4.0 & 2.7 & 22.0 & -6.8 & -9.1 \\
\hline Total basal area per stand $\left(\mathrm{m}^{2} / \mathrm{ha}\right)$ & 27 & 3.7 & 3.7 & 33 & -3.0 & -3.0 \\
\hline Mean volume per tree $\left(\mathrm{dm}^{3}\right)$ & 119 & 11.8 & 7.6 & 342 & -17.5 & -22.8 \\
\hline Total volume per stand ( $\left.\mathrm{m}^{3} / \mathrm{ha}\right)$ & 179 & 8.9 & 5.6 & 302 & -8.3 & -11.3 \\
\hline Total merchantable volume per stand $\left(\mathrm{m}^{3} / \mathrm{ha}\right)$ & 166 & 10.2 & 6.6 & 290 & -8.6 & -11.7 \\
\hline Total density per stand (stems/ha) & 1498 & -1.8 & -0.9 & 883 & 11.4 & 14.9 \\
\hline Relative stand density $(\% / 100)$ & 0.58 & 6.9 & 5.2 & 0.82 & -4.9 & -7.3 \\
\hline Total number of pulp logs per stand (logs/ha) & 2087 & 8.0 & 5.4 & 1791 & 7.5 & 20.6 \\
\hline Total number of saw logs per stand (logs/ha) & 103 & 42.7 & 25.2 & 1116 & -24.6 & -32.3 \\
\hline Total residual tip volume per stand $\left(\mathrm{m}^{3} / \mathrm{ha}\right)$ & 44.6 & 8.7 & 6.1 & 26.1 & 23.4 & 33.0 \\
\hline Total bark oven-dried biomass per stand ( $\mathrm{t} / \mathrm{ha}$ ) & 9.9 & 8.1 & 5.1 & 12.4 & -3.2 & -1.6 \\
\hline Total stem oven-dried biomass per stand ( $\mathrm{t} / \mathrm{ha})$ & 104.8 & 10.2 & 6.7 & 172.3 & -10.4 & -10.9 \\
\hline Total branch oven-dried biomass per stand (t/ha) & 9.0 & 8.9 & 5.6 & 17.8 & -14.6 & -15.2 \\
\hline Total foliage oven-dried biomass per stand (t/ha) & 5.5 & 7.3 & 3.6 & 9.3 & -11.8 & -11.8 \\
\hline Total above-ground oven-dried biomass per stand (t/ha) & 129.2 & 9.8 & 6.3 & 211.8 & -10.4 & -10.8 \\
\hline Total bark carbon per stand (t/ha) & 5.0 & 6.0 & 4.0 & 6.2 & -3.2 & -1.6 \\
\hline Total stem carbon per stand (t/ha) & 52.4 & 10.1 & 6.7 & 86.1 & -10.3 & -10.8 \\
\hline Total branch carbon per stand (t/ha) & 4.5 & 8.9 & 4.4 & 8.9 & -14.6 & -15.7 \\
\hline Total foliage carbon per stand ( $\mathrm{t} / \mathrm{ha})$ & 2.8 & 3.6 & 3.6 & 4.6 & -10.9 & -10.9 \\
\hline Total above-ground carbon per stand (t/ha) & 64.6 & 9.8 & 6.3 & 105.9 & -10.4 & -10.8 \\
\hline Total chip volume per stand-stud mill $\left(\mathrm{m}^{3} / \mathrm{ha}\right)$ & 65.0 & 10.0 & 6.5 & 101.3 & -9.2 & -9.6 \\
\hline Total lumber volume per stand—stud mill $\left(\mathrm{m}^{3} / \mathrm{ha}\right)$ & 69.0 & 14.9 & 9.4 & 170.9 & -18.3 & -20.5 \\
\hline Total chip volume per stand - randomized length mill $\left(\mathrm{m}^{3} / \mathrm{ha}\right)$ & 40.1 & 10.7 & 7.0 & 65.8 & -10.2 & -10.8 \\
\hline Total lumber volume per stand-randomized length mill $\left(\mathrm{m}^{3} / \mathrm{ha}\right)$ & 94.3 & 13.5 & 8.7 & 206.7 & -16.4 & -18.2 \\
\hline
\end{tabular}

a,b,c As defined in Table 3(a).

Table 4.

(a) Stand-level performance indices for jack pine plantations managed under a basic silvicultural intensity in northeastern Ontario by emission scenario and site quality. (b) Stand-level performance indices for jack pine plantations managed under a basic silvicultural intensity in northwestern Ontario by emission scenario and site quality.

(a)

\begin{tabular}{|c|c|c|c|c|c|c|}
\hline \multirow{2}{*}{$\begin{array}{l}\text { Index }^{\mathrm{a}} \\
\text { (Unit) }\end{array}$} & \multicolumn{6}{|c|}{ Site quality ${ }^{b}$} \\
\hline & \multicolumn{3}{|c|}{ Poor-to-medium } & \multicolumn{3}{|c|}{ Good-to-excellent } \\
\hline & \multicolumn{3}{|c|}{ Emission scenario $^{\mathrm{c}}$} & \multicolumn{3}{|c|}{ Emission scenario $^{\mathrm{c}}$} \\
\hline & $\mathrm{NC}$ & $\begin{array}{c}\text { B1 vs NC } \\
(\% \Delta)\end{array}$ & $\begin{array}{c}\mathrm{A} 2 \text { vs } \mathrm{NC} \\
(\% \Delta)\end{array}$ & $\mathrm{NC}$ & $\begin{array}{c}\text { B1 vs NC } \\
(\% \Delta)\end{array}$ & $\begin{array}{c}\mathrm{A} 2 \text { vs NC } \\
(\% \Delta)\end{array}$ \\
\hline Mean annual merchantable volume increment $\left(\mathrm{m}^{3} / \mathrm{ha} / \mathrm{yr}\right)$ & 3.8 & 0.0 & -7.9 & 6.3 & -14.3 & -23.8 \\
\hline Mean annual biomass increment ( $\mathrm{t} / \mathrm{ha} / \mathrm{yr})$ & 2.9 & 3.4 & -6.9 & 4.4 & -11.4 & -18.2 \\
\hline Mean annual carbon increment $(\mathrm{t} / \mathrm{ha} / \mathrm{yr})$ & 1.5 & 0.0 & -13.3 & 2.2 & -13.6 & -18.2 \\
\hline Percentage of sawlogs produced & 6.9 & 0.0 & -24.6 & 46 & -35.4 & -53.5 \\
\hline Lumber volume recovered—stud mill (\%) & 53.1 & 0.0 & -2.3 & 64 & -5.2 & -8.6 \\
\hline Lumber volume recovered—randomized length mill (\%) & 70.9 & 0.0 & -0.8 & 76.6 & -2.5 & -3.9 \\
\hline Land expectation value—stud mill (CAN\$K/ha) & 1.0 & 2.3 & -51.2 & 7.7 & -42.4 & -61.3 \\
\hline Land expectation value - randomized length mill (CAN\$K/ha) & 3.1 & 1.2 & -21.5 & 10.1 & -33 & -48 \\
\hline Duration of optimal site occupancy ( $\%$ of rotation) & 28 & -14.3 & 0.0 & 16 & -25.0 & 0.0 \\
\hline Mean height/diameter ratio $(\mathrm{m} / \mathrm{m})$ & 88.9 & 0.6 & 0.8 & 86.4 & 1.9 & 1.3 \\
\hline Mean wood density $\left(\mathrm{g} / \mathrm{cm}^{3}\right)$ & 0.4556 & -0.2 & 0.2 & 0.4461 & -0.7 & -0.6 \\
\hline Mean maximum branch diameter $(\mathrm{cm})$ & 2.86 & -0.7 & -0.3 & 2.80 & -1.1 & -1.8 \\
\hline Time to operability status (yr) & 44 & 0.0 & 2.3 & 38 & 5.3 & 15.8 \\
\hline Time of initial crown closure (yr) & 13 & 23.1 & 23.1 & 9 & 22.2 & 11.1 \\
\hline
\end{tabular}

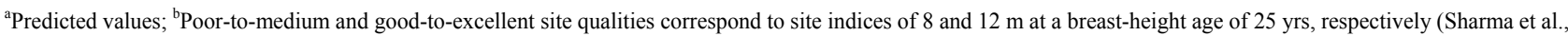
2012). ${ }^{\mathrm{c}}$ As defined in the text. 


\section{P. F. NEWTON}

(b)

\begin{tabular}{|c|c|c|c|c|c|c|}
\hline \multirow{4}{*}{$\begin{array}{l}\text { Index }^{a} \\
\text { (Unit) }\end{array}$} & \multicolumn{6}{|c|}{ Site quality $^{\mathrm{b}}$} \\
\hline & \multicolumn{3}{|c|}{ Poor-to-medium } & \multicolumn{3}{|c|}{ Good-to-excellent } \\
\hline & \multicolumn{3}{|c|}{ Emission scenario $^{\mathrm{c}}$} & \multicolumn{3}{|c|}{ Emission scenario $^{c}$} \\
\hline & $\mathrm{NC}$ & $\begin{array}{c}\text { B1 vs NC } \\
(\% \Delta)\end{array}$ & $\begin{array}{c}\mathrm{A} 2 \text { vs NC } \\
(\% \Delta)\end{array}$ & $\mathrm{NC}$ & $\begin{array}{c}\text { B1 vs NC } \\
(\% \Delta)\end{array}$ & $\begin{array}{c}\mathrm{A} 2 \text { vs NC } \\
(\% \Delta)\end{array}$ \\
\hline Mean annual merchantable volume increment $\left(\mathrm{m}^{3} / \mathrm{ha} / \mathrm{yr}\right)$ & 3.3 & 12.1 & 6.1 & 5.8 & -8.6 & -12.1 \\
\hline Mean annual biomass increment (t/ha/yr) & 2.6 & 7.7 & 3.8 & 4.2 & -9.5 & -9.5 \\
\hline Mean annual carbon increment (t/ha/yr) & 1.3 & 7.7 & 7.7 & 2.1 & -9.5 & -9.5 \\
\hline Percentage of sawlogs produced & 4.7 & 29.8 & 17.0 & 38.4 & -20.8 & -32.6 \\
\hline Lumber volume recovered—stud mill (\%) & 51.5 & 2.1 & 1.4 & 62.8 & -4.0 & -4.9 \\
\hline Lumber volume recovered—randomized length mill (\%) & 70.2 & 0.7 & 0.4 & 75.9 & -1.8 & -2.2 \\
\hline Land expectation value—stud mill (CAN\$K/ha) & 0.3 & 137.1 & 84.3 & 6.4 & -37.1 & -38.8 \\
\hline Land expectation value - randomized length mill (CAN\$K/ha) & 2.2 & 28.6 & 18.2 & 8.8 & -28.1 & -28.9 \\
\hline Duration of optimal site occupancy (\% of rotation) & 32 & -12.5 & -12.5 & 16 & -25.0 & 0.0 \\
\hline Mean height/diameter ratio (m/m) & 89.1 & -1.0 & -0.4 & 86.9 & 0.2 & 0.5 \\
\hline Mean wood density $\left(\mathrm{g} / \mathrm{cm}^{3}\right)$ & 0.4577 & -0.4 & -0.3 & 0.4460 & -0.7 & -0.6 \\
\hline Mean maximum branch diameter $(\mathrm{cm})$ & 2.87 & -1.0 & -0.7 & 2.80 & -1.4 & -1.4 \\
\hline Time to operability status (yr) & 46 & -4.3 & -4.3 & 39 & 5.1 & 7.7 \\
\hline Time of initial crown closure (yr) & 12 & 25.0 & 25.0 & 8 & 25.0 & 25.0 \\
\hline
\end{tabular}

${ }_{\text {a,b,c }}$ As defined in Table 4(a).

\section{Higher Site Qualities}

The effects of climate change were clearly evident for the stands managed on the good-to-excellent site qualities irrespective of locale. The size-density trajectories of the stands grown under the NC scenario incurred greater mortality during the middle of the rotation whereas the stands grown under the B1 and A2 exhibited greater rates of mortality later in the rotation (Figures 1(b) and 2(b)). The stands grown under the B1 and A2 scenarios illustrated a continuous decline in the rate of their stand development as measured by their size-density trajectories and dominant height status during the later stages of the rotation (Figures 1(b) and 2(b)). Relative to the NC scenario, rotational mean volumes were approximately $33 \%$ smaller within the northeastern stands grown under the B1 and A2 scenarios (Table 3(a)). Similarly, for the northwestern stands, mean volumes were on average $20 \%$ smaller relative to the NC scenario (Table 3(b)). In terms of development, the dominant height of the northeastern stands grown under the B1 and A2 scenarios were on average $11 \%$ smaller at rotation than that obtained within the stand grown under the NC scenario (Table 3(a)). Likewise, for the northwestern stands, the dominant heights at rotation were on average $13 \%$ smaller within the stands grown under the $\mathrm{B} 1$ and $\mathrm{A} 2$ scenarios, relative to the stand grown under the NC scenario (Table 3(b)).

Volumetric productivity declined by $14 \%$ and $24 \%$ within the northeastern stands grown under the B1 and A2 scenarios, respectively, compared to the stand grown under the $\mathrm{NC}$ scenario (Table 4(a)). The corresponding values for the northwestern stands were $9 \%$ and $12 \%$ for the B1 and A2 scenarios, respecttively (Table 4(b)). Biomass production and carbon yields exhibited similar levels of decline for both the B1 and A2 scenarios, in both regions (Tables 4(a) and (b)). Relative to the stands grown under the NC scenario, the proportion of preferred endproducts (number of sawlogs and volume of dimensional lumber) and associated economic values at the time of harvest (land expectation value), declined within the stands grown under the
B1 and A2 scenarios, irrespective of locale (Tables 4(a) and (b)). Similar to the results from the low quality sites, differences in the duration of optimal site occupancy, structural stability (height/diameter ratio), wood quality metrics, and time to crown closure, were minimal among all three scenarios (Tables 4(a) and (b)). In terms of operability, however, the northeastern stands grown under the B1 and A2 scenarios required an additional 2 and 6 years, respectively, to attain operability status. Corresponding values for the northwestern stands were 2 and 3 years, respectively.

\section{Discussion}

The results of this study suggest that the predicted warmer temperatures and wetter growing seasons arising from increases in the emission of greenhouse gases and aerosols will negatively affect jack pine productivity on good-to-excellent site qualities over the next 50 years (2011-2061). Specifically, stands managed employing a basic silvicultural intensity (Bell et al., 2008) and grown on such sites under either a B1 or A2 scenario will experience declines in their rate of development. By the end of the rotation, these declines will result in reductions in rotational mean sizes (11\% for quadratic mean diameter and $27 \%$ for mean volume) and per unit area yields (14\% for total volume and merchantable volumetric yields, and $13 \%$ for biomass production and carbon outcomes), recoverable end-product volumes $(13 \%$ for the volume of recoverable chip and $23 \%$ for lumber volumes), economic worth ( $40 \%$ for land expectation value), and operability status ( $8 \%$ longer to attain the threshold operability targets), relative to comparable stands grown under the NC scenario. Contrary to expectation, these results suggest that the warmer temperatures combined with increases in precipitation forecasted under the B1 and A2 scenarios may actually degrade the productivity of jack pine plantations. Although speculative, one plausible explanation is that this additional moisture will not be made available due to the low moisture retention 


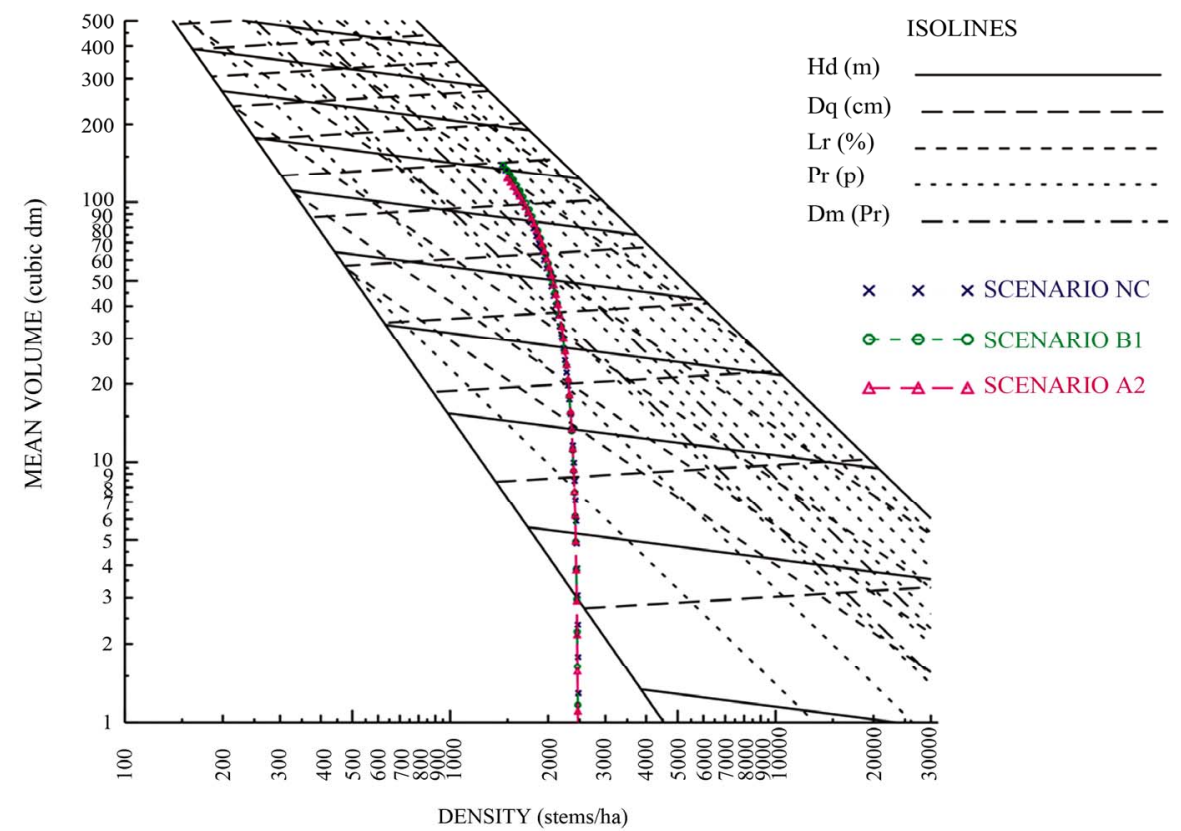

(a)

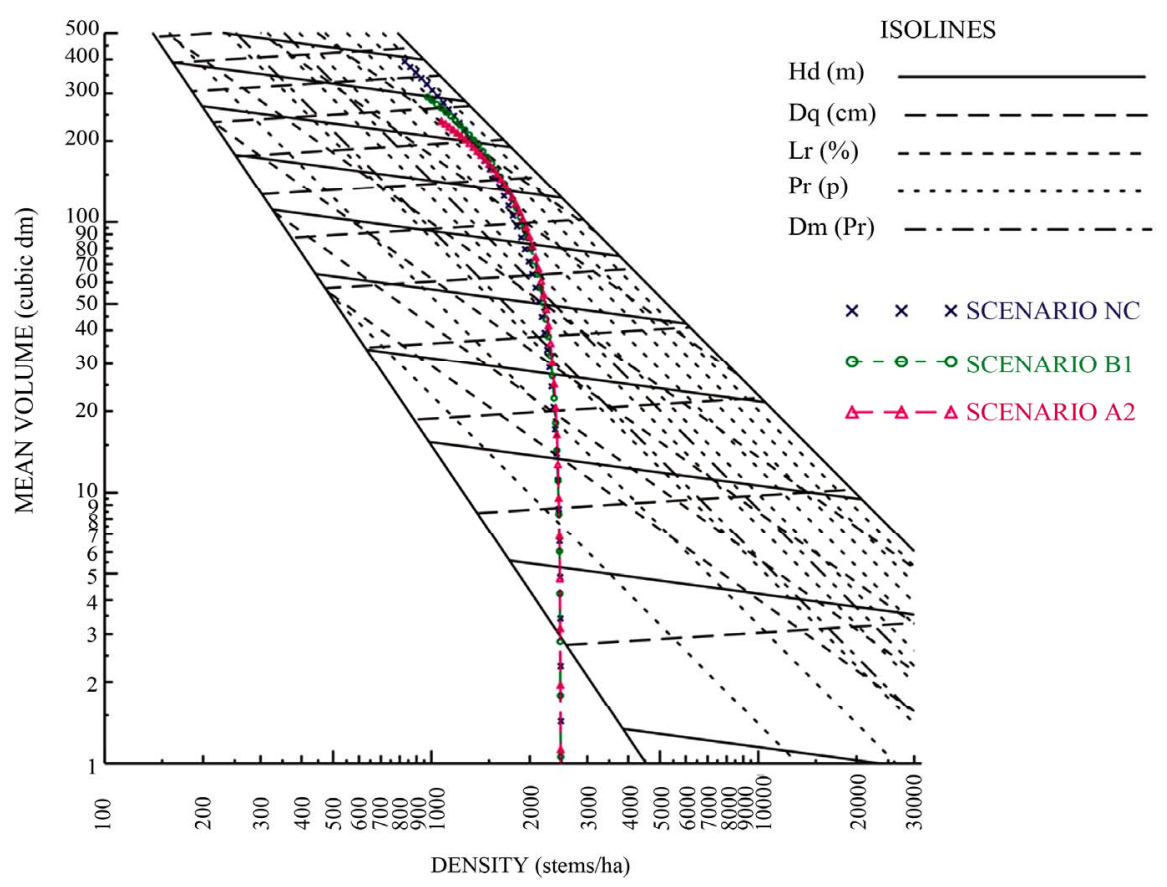

(b)

Figure 1.

(a) Temporal size-density trajectories by scenario for jack pine plantations managed under a basic silvicultural intensity as presented within the traditional stand density management diagram (SDMD) graphic for a low-to-medium quality site $\left(S_{I}=8\right)$ situated in northeastern Ontario. Graphically illustrating: 1) isolines for mean dominant height (Hd; $4-22 \mathrm{~m}$ by $2 \mathrm{~m}$ intervals proceeding vertically upwards), quadratic mean diameter (Dq; $4-26 \mathrm{~cm}$ by $2 \mathrm{~cm}$ intervals proceeding vertically upwards), mean live crown ratio ( $\mathrm{Lr} ; 35 \%, 40 \%, 50 \%, \cdots, 80 \%$ proceeding from left-to-right horizontally), relative density index ( $\operatorname{Pr} ; 0.1-1.0$ by 0.1 intervals proceeding left-to-right horizontally); 2 ) crown closure line (lower diagonal solid line) and self-thinning rule at a $\mathrm{Pr}=1.0$ (upper diagonal solid line); 3 ) lower and upper $\operatorname{Pr}$ values delineating the optimal density management window (Dm; $0.32 \leq \operatorname{Pr} \leq 0.45$ ); and 4) expected 50 year size-density trajectories with 1 year intervals denoted for each scenario. (b) Temporal size-density trajectories by scenario for jack pine plantations managed under a basic silvicultural intensity as presented within the SDMD graphic for a good-to-excellent quality site $\left(S_{I}=12\right)$ situated within northeastern Ontario. Graphical denotations as defined in Figure 1(a). 


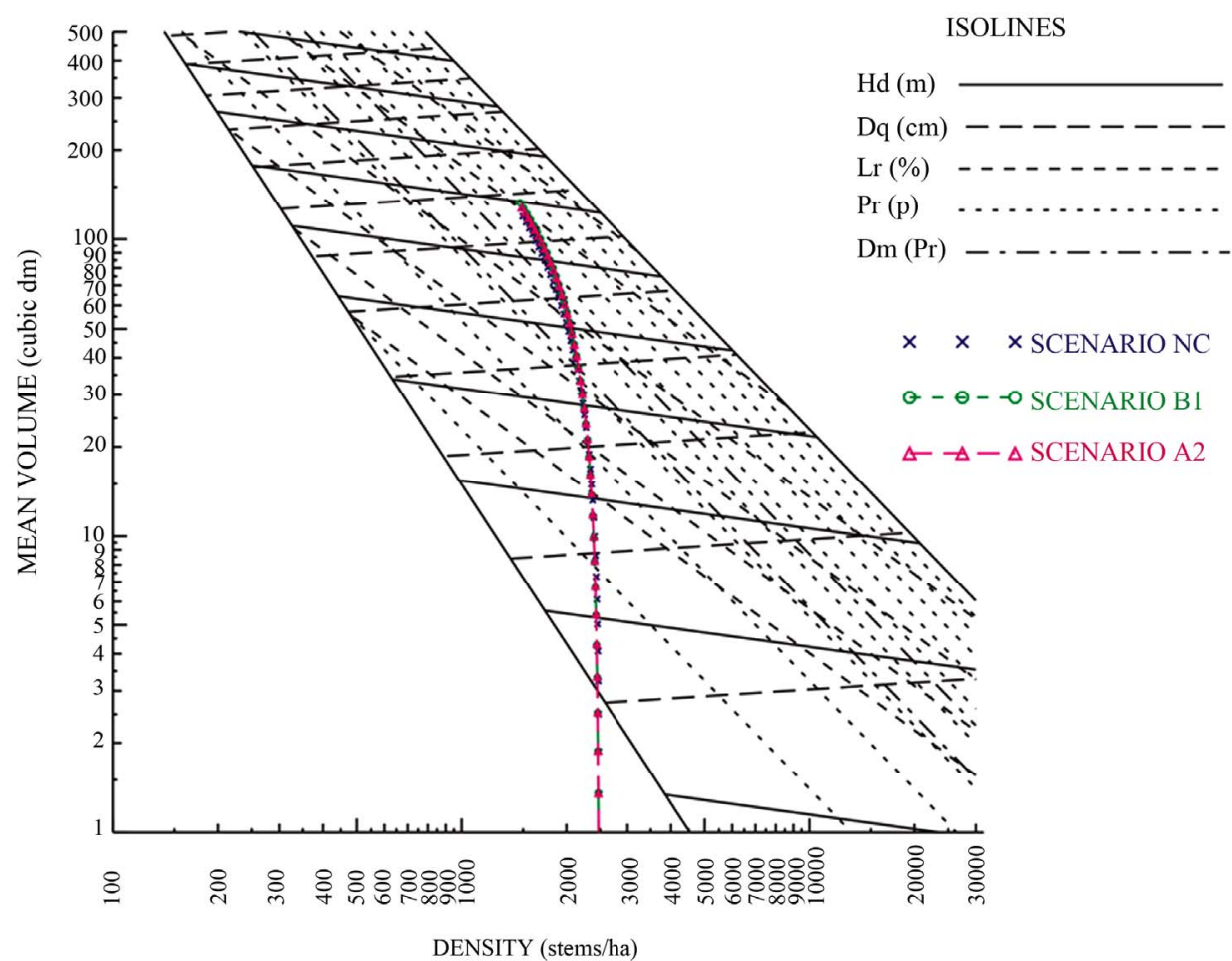

(a)

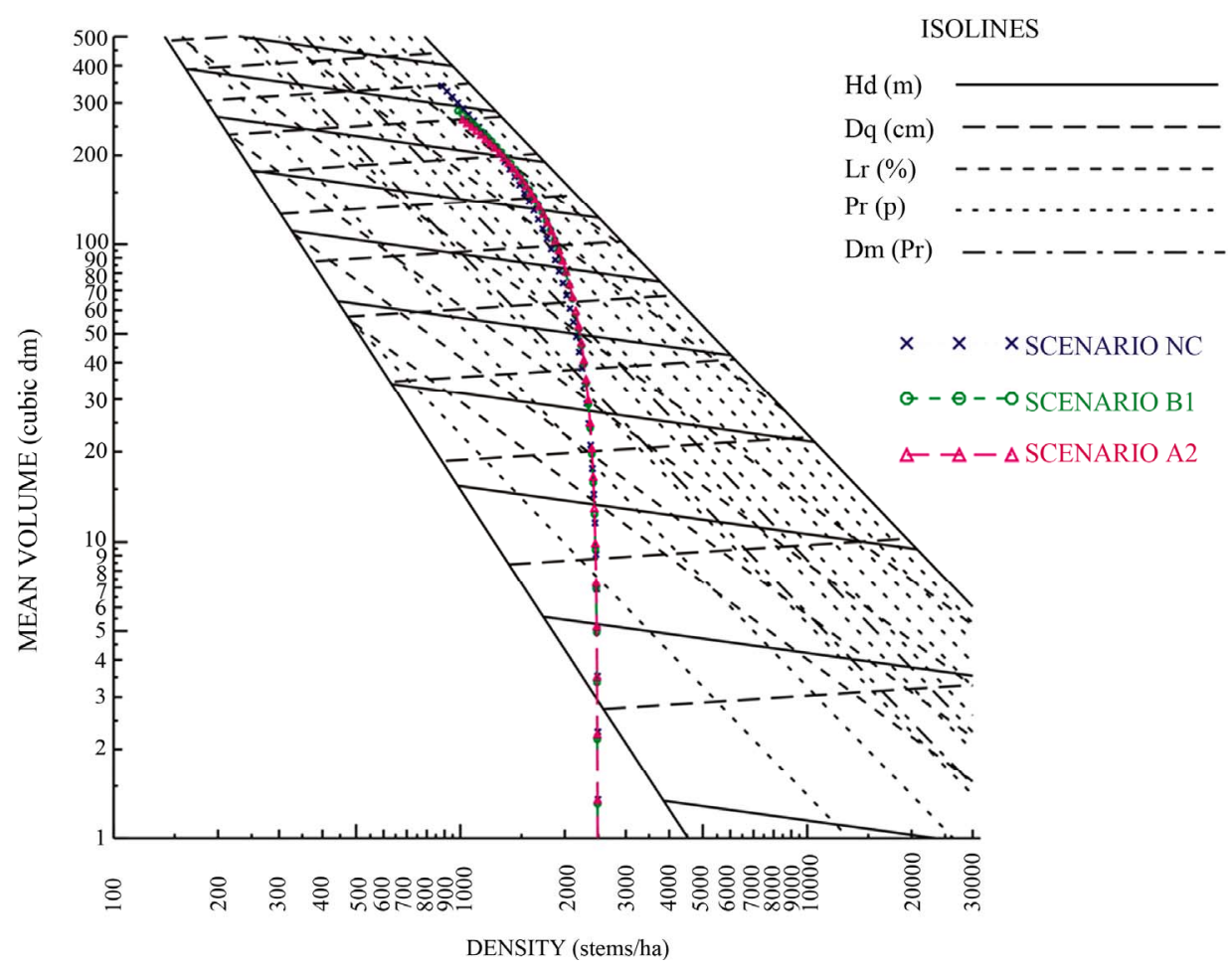

(b)

Figure 2.

(a) Temporal size-density trajectories by scenario for jack pine plantations managed under a basic silvicultural intensity as presented within the SDMD graphic for a low-to-medium quality site $\left(S_{I}=8\right)$ situated within northwestern Ontario. Graphical denotations as defined in Figure 1(a); (b) Temporal size-density trajectories by scenario for jack pine plantations managed under a basic silvicultural intensity as presented within the SDMD graphic for a good-to-excellent quality site $\left(S_{I}=12\right)$ situated within northwestern Ontario. Graphical denotations as defined in Figure 1(a). 
ability of the sites that jack pine traditionally occupies. Additionally, temperature-induced increases in the rates of evapotranspiration and tree respiration (Boisvenue \& Running, 2006) during the growing season may divert some of the photosynthate resources away from the production of new plant tissue.

Conversely, the productivity of jack pine plantations established on poor-to-medium site qualities may be somewhat invariant to the forecasted changes in climate, depending on a stand's specific geographical location. Mensurational-based yield and performance metrics for the northeastern stands grown under the $\mathrm{NC}$ and $\mathrm{B} 1$ scenarios were approximately equivalent to that of the stand grown under the A2 scenario. The northwestern stands grown under the B1 and A2 scenarios actually benefitted from the increase in temperature and precipitation as evident by the slightly improved mean tree size and per unit area yield outcomes. Ecologically, this may indicate that jack pine plantations situated on poor-to-medium quality sites at the northwestern locale may be moisture deficient. Thus these stands may be able to gain from a more maritime-like climate in the future. Notably, across both site quality classes assessed and irrespective of locale, effects arising from either the B1 or A2 scenario did not appreciatively affect the duration of optimal site occupancy, stand stability or wood quality metrics.

The site-specific patterns observed in this study suggest that jack pine productivity will be affected to a much greater degree on the higher site qualities than on the lower site qualities. Similar general trends were reported by Loustau et al. (2005) who found that climate change effects on the productivity of forests in western Europe were greatest on high fertility sites.

The importance of scaling global climate change effects to the local level is increasingly being acknowledged within the climate change literature (e.g., Malone \& Engle, 2011). Combining site-specific future estimates of climatic variables under various emission scenarios with a forest productivity model, as shown in this study, readily facilitates the evaluation of climate change effects at the local level. The two regions selected for assessment in this study reflected representative examples within two of the most important areas for jack pine management in Ontario. These areas were geographically separated and inherently different in terms of their biophysical characteristics. Consequently, the results reported in this study represent a plausible range of outcomes for this particular species within the central portion of its range.

\section{Modeling Approach and Associated Limitations}

The objective of this study was to evaluate the potential effect of projected future climate conditions on the productivity of jack pine plantations over the next 50 years (2011-2061). Analytically, climatic parameters as predicted by a global climate model in association with a regional spatial climatic model, under 3 plausible emissions scenarios, were used as input values to a biophysical-based site-specific height-age function. The resultant function when integrated within the CROPLANNER simulation model enabled the estimation of climate change effects on a broad array of stand-level productivity measures. A similar approach was used to predict the effects of climate change on lodgepole pine (Pinus contorta Douglas ex Louden) in British Columbia (O’Neill \& Nigh, 2011). In this case, the site-height equation within the Tree and Stand Simulator (TASS) was modified in order to account for changing climatic conditions for specific genotypes and locales. Ninety-year simulations for three disparate provenances growing under three emission sce- narios suggested that volumetric yields would decline by $7 \%$ $13 \%$. Although acknowledging differences in species, scenarios, locale, site qualities and models between the studies, the trend of decrease productivity with future climate change is projected for both of these pine species. However, similar to most attempts to model the effects of climate change, the validity of future longterm forecasts is unknown and hence caution must be exercised when interpreting the projected consequences, irrespective of the modeling approach utilized.

The approach utilized in this study reinforces the utility of modifying existing empirical-based model structures in order to account for climate change effects on yield outcomes. However, the approach assumes that the wide array of biological changes arising from climate change can be expressed through the sitebased height-age equation. Although height growth is the uni- versally accepted driver of forest productivity (O'Neill \& Nigh, 2011), this is nevertheless a simplifying assumption which requires further verification. For example, a warmer and wetter climate may increase growth losses due to biotic (insect and disease) and abiotic (wind) agents (e.g., Fleming, 2000). The effects of increased decomposition rates and the $\mathrm{CO}_{2}$ fertilization effect will also affect productivity (e.g., Girardin et al., 2011). Consequently, the effects on the insect and disease vectors and changes in the hydrological and biochemical cycles are not directly addressed using this approach. Hence until these effects of climate change are better understood, the results of this study should be considered tentative. Nevertheless, the initial projections and associated inferences suggest that jack pine may respond in site specific manner with higher site qualities experiencing the largest declines. Therefore, current yield projections derived from models that do not account for climate change effects are likely over-estimating future productivity on the better site qualities.

The scenarios evaluated in this study consisted of a static scenario which was composed of climatic variables not changing from their historical norms, the conceptual B1 emission scenario based on convergent greener world characterized by collective approaches to solving economic, social, and environmental problems, and the A2 scenario which arises from a decentralized and heterogeneous organized world where collective initiatives for addressing global environmental problems are minimal. However, the consequences of these scenarios were only assessed for the 2011-2061 period and thus included only 2 of the 3 projection periods normally considered under long-term climate change modeling (i.e., 2011-2040, 2041-2070 and 20712100). Consequently, the scope of the simulations did not include the most severe period of predicted climate change and hence the results do not reflect the full potential of the negative ramifications arising from extreme changes in climate on jack pine productivity. Forest managers and policy makers will need to account for these more severe climate change effects when forecasting the future development of plantations managed under a 50 yr rotation that are established after 2020.

\section{Conclusion}

The simulation results from the CROPLANNER model indicated that future yields for stands situated on low-to-medium quality sites were largely unaffected by the predicted increased temperature and precipitation rates during the 2011-2061 period. Conversely, however, stands situated on good-to-excellent quality sites grown under the B1 and A2 scenarios experienced consequential declines in stand development: reductions of $6.6 \%$ and $12.0 \%$ in mean dominant height at rotation for the $\mathrm{B} 1$ and $\mathrm{A} 2$ scenarios, 
respectively, relative to the NC scenario. These declines translated into decreases in merchantable volume productivity (mean annual merchantable volume increment) in the order of $19 \%$ for the northeastern stands and $10 \%$ for the northwestern stands grown under the B1 and A2 scenarios. Similar declining trends were evident for rotational mean sizes (quadratic mean diameter and mean stem volume), biomass yields (component-specific biomass production and carbon outcomes), recoverable end-product volumes (volume of recoverable chip and lumber volumes), and economic worth (land expectation value). In addition to providing plausible site-specific climate change outcomes on jack pine productivity within the central portion of the species range, the results suggest that future predictions made under the no change emission scenario may overestimate merchantable volume productivity on the higher site qualities by as much as $15 \%$. Consequently, forest managers should exercise caution when interpreting future long-term yield forecasts derived from models that have yet to account for climate change effects.

\section{Acknowledgements}

The author expresses his appreciation to: 1) Dr. Sharma, Research Scientist, Ontario Forest Research Institute, Ontario Ministry of Natural Resources, Sault Ste. Marie, Ontario, Canada, for providing assess to the biophysical height-age function; 2) John Parton, Provincial Growth and Yield Modeler, Ontario Ministry of Natural Resources, South Porcupine, Ontario, Canada, for provision of constructive input during the early phase of this study; and 3) to the anonymous reviewers for their constructive comments and suggestions.

\section{REFERENCES}

Bell, W. F., Parton, J., Stocker, N., Joyce, D., Reid, D., Wester, M., Stinson, A., Kayahara, G., \& Towill. B. (2008). Developing a silvicultural framework and definitions for use in forest management planning and practice. Forestry Chronicle, 84, 678-693.

Boisvenue, C., \& Running, S. W. (2006). Impacts of climate change on natural forest productivity-Evidence since the middle of the 20th century. Global Change Biology, 12, 1-21.

Carmean, W. H., Niznowski, G. P., \& Hazenberg, G. (2001). Polymorphic site index curves for jack pine in Northern Ontario. Forestry Chronicle, $77,141-150$.

Colombo, S. J., McKenney, D. W., Lawrence, K. M., \& Gray, P. A. (2007). Climate change projections for Ontario: A practical guide for policymakers and planners. Ontario Ministry of Natural Resources, Applied Research Development Branch, Peterborough Ontario. Climatic Change Research Report CCRR-05.

Environment Canada (2011). The third generation coupled global climate model. URL (last checked 15 July 2011) http://www.ec.gc.ca/ccmac-cccma/default.asp?lang=En\&n=1299529 F-1

Fleming, R. A. (2000). Climate change and insect disturbance regimes in Canada's boreal forests. World Resource Review, 12, 520-555.

Girardin, M. P., Raulier, F., Bernier, P. Y., \& Tardif, J. C. (2008). Response of tree growth to a changing climate in boreal central Canada: A comparison of empirical, process-based, and hybrid modeling approaches. Ecological Modelling, 213, 209-228. doi:10.1016/j.ecolmodel.2007.12.010

Girardin, M. P., Bernier, P. Y., Raulier, F., Tardif, J. C., Conciatori, F., \& Guo, X. J. (2011). Testing for a $\mathrm{CO}_{2}$ fertilization effect on growth of Canadian boreal forests. Journal of Geophysical Research, 116, 1-16. doi:10.1029/2010JG001287

IPCC (Intergovernmental Panel on Climate Change) (2007). Climate change 2007: Synthesis report. Contribution of working groups I, II and III to the fourth assessment report of the Intergovernmental
Panel on Climate Change. Geneva: IPCC.

Ise, T., \& Moorcroft, P. R. (2010). Simulating boreal forest dynamics from perspectives of ecophysiology, resource availability, and climate change. Ecological Research, 25, 501-511. doi:10.1007/s11284-009-0680-8

Kurz, W. A., Dymonda, C. C., White, T. M., Stinson, G., Shaw, C. H., Rampley, G. J., Smytha, C., Simpson, B. N., Neilson, E. T., Trofymow, J. A., Metsaranta, J., \& Apps, M. J. (2009). CBM-CFS3: A model of carbon-dynamics in forestry and land-use change implementing IPCC standards. Ecological Modeling, 220, 480-504. doi:10.1016/j.ecolmodel.2008.10.018

Loustau, D., Bosc, A., Colin, A., Ogée, J., Davi, H., François, C., Dufrêne, E., Déqué, M., Cloppet, E., Arrouays, D., Le Bas, C., Saby, N., Pignard, G., Hamza, N., Granier, A., Bréda, N., Ciais, P., Viovy, N., \& Delage, F. (2005). Modeling climate change effects on the potential production of French plains forests at the sub-regional level. Tree Physiology, 25, 813-823.

Malone, E., \& Engle, N. (2011). Evaluating regional vulnerability to climate change: Purposes and methods. WIRES Climatic Change, 2, 462-474. doi:10.1002/wcc. 116

McKenney, D., Papadopol, P., Campbell, K., Lawrence, K., \& Hutchinson, M. (2006). Spatial models of Canada- and North America-wide 1971/2000 minimum and maximum temperature, total precipitation and derived bioclimatic variables. Canadian Forest Service, Great Lakes Forestry Centre, Sault Ste. Marie, Ontario. Frontline Technical Note No. 106.

Myneni, R. B., Keeling, C. D., Tucker, C. J., Asrar, G., \& Nemani, R. R. (1997). Increased plant growth in the northern high latitudes from 1981 to 1991. Nature, 386, 698-702. doi:10.1038/386698a0

Nakicenovic, N., Alcamo, J., Davis, G., Vries, B. D., Fenhann, J., Gaffin, S., Gregory, K., Grübler, A., Jung, T. Y., Kram, T., Rovere, E. L. L., Michaelis, L., Mori, S., Morita, T., Pepper, W., Pitcher, H., Price, L., Riahi, K., Roehrl, A., Rogner, H.-H., Sankovski, A., Schlesinger, M., Shukla, P., Smith, S., Swart, R., Rooijen, S. V., Victor, N., \& Dadi, Z. (2000). IPCC Special Report on Emissions Scenarios (SRES). New York, NY: Cambridge University Press.

Newton, P. F. (2009). Development of an integrated decision-support model for density management within jack pine stand-types. Ecological Modelling, 220, 3301-3324. doi:10.1016/j.ecolmodel.2009.07.025

OMNR (Ontario Ministry of Natural Resources) (2008). Annual report on forest management 2006/07. URL (last checked August 10, 2011) http://www.web2.mnr.gov.on.ca/mnr/forests/public/publications/Ann ual\%20Reports/2006-2007/English/AR06_07_file_one.pdf

O'Neill, G. A., \& Nigh, G. (2011). Linking population genetics and tree height growth models to predict impacts of climate change on forest production. Global Change Biology, 17, 3208-3217. doi:10.1111/j.1365-2486.2011.02467.x

Parker, W. C., Colombo, S. J., Cherry, M. L., Greifenhagen, S., Papodopol, C., Flannigan, M. D., McAlpine, R. S., \& Scarr, T. (2000). Third millennium forestry: What climate change might mean to forests and forest management in Ontario. Forestry Chronicle, 76, 445-463.

Rowe, J. S. (1972). Forest regions of Canada. Government of Canada, Department of Environment, Canadian Forestry Service, Ottawa, Ontario. Publication No. 1300.

Rudolf, R. D., \& Yeatman, C. W. (1982). Genetics of jack pine (Pinus banksiana). Government of the United States of America, Department of Agriculture, Forest Service, Lake States Forest Experimental Station, St. Paul, Minnesota, Research Paper WO-38.

Sharma, M., Subedi, N., Ter-Mikaelian, M., \& Parton, J. (2012). Modeling stand height/site index of plantation grown jack pine and black spruce trees in a changing climate. In preparation.

Shaw, C., Chertov, O., Komarov, A., Bhatti, J., Nadporozskaya, M., Apps, M., Bykhovets, S., \& Mikhailov, A. (2006). Application of the forest ecosystem model EFIMOD 2 to jack pine along the Boreal Forest Transect Case Study. Canadian Journal of Soil Science, 86, 171-185. doi:10.4141/S05-079

Zhang, S. Y., \& Koubaa, A. (2008). Softwoods of eastern Canada: Their silvics characteristics, manufacturing and end-uses. Special Publication SP-526E, FPInnovations, Forintek. 